Proceedings of XIX International Scientific Conference "New Technologies and Achievements in Metallurgy, Material Engineering, Production Engineering and Physics", Częstochowa, Poland, June 7-8, 2018

\title{
Research on Thermal Contact Resistance in a Bed of Steel Square Bars Using Thermovision
}

\author{
T. WYLECIAE ${ }^{a, *}$ AND D. URBANIAK ${ }^{b}$ \\ ${ }^{a}$ Department of Industrial Furnaces and Environmental Protection, Czestochowa University of Technology, \\ al. Armii Krajowej 19, 42-200 Czestochowa, Poland \\ ${ }^{b}$ Institute of Thermal Machinery, Czestochowa University of Technology, \\ al. Armii Krajowej 21, 42-200 Czestochowa, Poland
}

\begin{abstract}
The article presents the results of experimental research on the determination of thermal resistance values occurring on contact surfaces of adjacent steel square bars. The places of such contact in the theory of contact heat conduction are called connectors. More generally, this problem is related to the determination of the effective thermal conductivity of the porous charge in the form of a bundle of square bars. The value of coefficient $k_{e f}$ is necessary to optimize the heat treatment of square bars heated in the form of bundles. One of the most important parameters affecting the value of $k_{e f}$ is the thermal resistance of the connectors. This size of this can be determined both analytically and experimentally. Due to the geometrical complexity of the connectors being created by surfaces of adjacent bars and the oxidation phenomenon, the theoretical models developed so far cannot be applied. On the other hand, in standard measurement techniques of this parameter the sample with a length of several centimeters are used, which allows measurement of the temperature distribution. Because the transverse dimensions of bars are an order of magnitude smaller, these methods cannot be used in the analyzed case, too. It was decided to use a partially innovative experimental technique to determine the considered thermal resistance. The originality of the proposed method consists in using infrared measurements to research the temperature field of the analyzed medium. On the basis of the obtained data, the value of the sought parameter will be determined using the analytical dependences concerning heat conduction.
\end{abstract}

DOI: 10.12693/APhysPolA.135.263

PACS/topics: porous charge, thermal contact resistance, thermovision, effective thermal conductivity

\section{Introduction}

In technical processes related to the production or operation of various production devices in which heat exchange takes place, contact conductivity of heat is very common. It is a method of heat transfer between adjoining surfaces of solids. The following industries are most often given as examples of the occurrence of this phenomenon: cryogenics, space technologies, aviation, nuclear energy, nanotechnologies, and microelectronics $[1,2]$. Contact heat transfer also plays an important role in various processes involved in the manufacturing and processing of steel products. Most often these are continuous steel casting and plastic forming processes [3, 4].

Another type of steel products processing, in which the contact conductivity of heat plays an important role in their course, is heat treatment. This is the case when heating a charge with a porous structure. This category includes beams of various types of long elements (e.g. rods, rectangular profiles, pipes) and coils of steel or wire [5]. The most characteristic examples of steel porous charge in the form of bundles of bars and wire coils are shown in Fig. 1. Due to the lack of continuity of the solid phase of the porous charge, its heating

\footnotetext{
* corresponding author
}

in the heat treatment processes is quite differently from the heating of solid elements. Mainly the phenomenon of contact heat conduction occurring in places of contact of adjacent bars in bundles or coils of wire is responsible for the differentness of the mentioned processes.

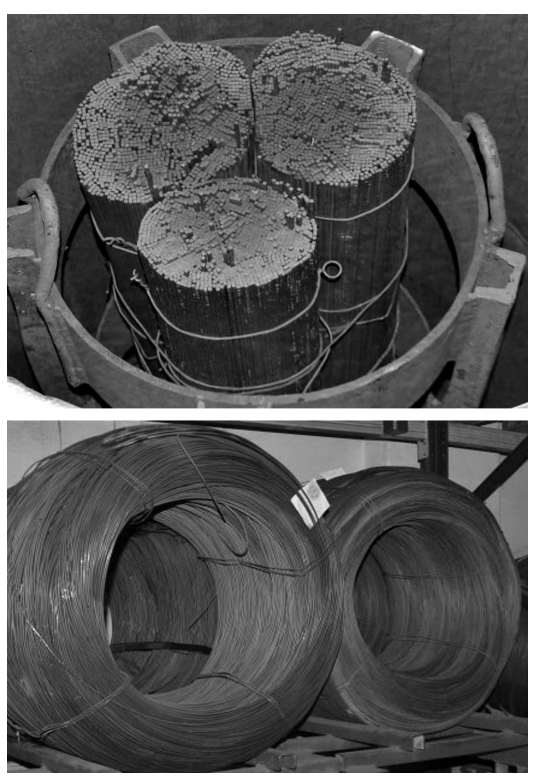

Fig. 1. Bar bundles and wire coils as most characteristic examples of porous charge. 
The heat transfer in the volume of the porous charge as a bundle of bars or wire coils is a combination of the following physical phenomena: conduction in the crosssection of individual elements, conduction in the gas that fills free spaces of the charge, contact conductivity in the places of contact of adjacent elements, and thermal radiation between their surfaces. Due to the complexity of this phenomenon, its course is influenced by a lot of different factors, i.e. temperature and porosity of the charge, thermal conductivity of steel and gas, shape and surface condition of individual elements forming this charge.

A significant number of factors affecting intensity of heat transfer in the area of the porous charge makes the numerical simulation of this process a complicated case. One of the methods used to simplify computational models is to use the concept of effective thermal conductivity $k_{e f}[6,7]$. This size is widely used in the theory of porous media and quantifies the complex heat transfer by analogy to the thermal conduction coefficient in classical conduction $[8,9]$. Thanks to this approach, the actual heat flow in the volume of the porous charge is treated as heat conduction in a homogeneous material. This makes it possible to significantly simplify the mathematical description of very complex heat transfer processes.

However, determining the coefficient value $k_{e f}$ and especially its changes in the temperature function for a given porous charge is not a simple issue. One of the methods of modelling effective thermal conductivity is the analysis of thermal resistance. In this approach, for a representative part of the analyzed medium, a corresponding thermal resistance is assigned to each heat exchange method. Based on a combination of serial and parallel connections of these resistances, the total thermal resistance $R_{t o}$ is determined. Knowing this size, effective thermal conductivity of the charge is calculated from equation describing conduction thermal resistance of flat bed [10]. The application of this methodology to determine the coefficient $k_{e f}$ for a given porous charge requires knowledge about the resistance of contact conduction. The article presents the original method of experimental determination of $R_{t o}$ resistance using the thermovision technique. The presented research was carried out for a porous charge made of $20 \mathrm{~mm}$ square bars. Due to the geometry of the test stand, the sample of the tested charge was in the form of a flat bed.

\section{Analysis and measurements}

In the theory of contact heat conduct, the contact area of two surfaces is called a joint. Since the surfaces of real bodies are never perfectly smooth, the direct contact of adjoining elements occurs in the joint only on a small part of the nominal surface $A_{n}$, called the actual contact surface $A_{r}$. The size of the $A_{r}$ surface depends on the mechanical load of the joint and the deformation of the roughness tops and it is the sum of a large number of discrete microcontacts [2]. The quotient of the actual contact surface to the nominal surface of the joint is called the contact ratio or relative real contact area and denotes as $\phi_{c t}$.

For the determination of thermal contact resistance, both analytical and experimental methods are used. To calculate this parameter, equations are used, the form of which depends on the adopted model of surface deformation of microcontacts. Depending on the value of the pressing stress $P$ present in the joint, one of the following deformation models is used: elastic, plastic, or elastic-plastic [11]. On this basis, three basic parameters describing the geometric properties of the joint are calculated: contact ratio $\phi_{c t}$, density of contact spots $n_{c t}$ and mean radius of contact spots $r_{c t}$. For the three deformation models mentioned above, the general dependence for calculating resistance $R_{c t}$, which is in the form [12], was established

$$
R_{c t}=\frac{\left(1-\phi_{c t}^{0,5}\right)^{1,5}}{2 n_{c t} r_{c t} k_{m}},
$$

where $k_{m}$ is the effective conductivity of the joint

$$
k_{m}=\frac{2 k_{1} k_{2}}{k_{1}+k_{2}} .
$$

Specific formulae for calculating individual parameters occurring in Eq. (1) depending on the adopted model of the deformation can be found in [13, 14]. For example, for the plastic deformation model, the geometric parameters of the joint occurring in Eq. (1) are described as follows:

$$
\begin{aligned}
& \phi_{c t}=0.5 \operatorname{erfc}\left(\frac{\lambda}{\sqrt{2}}\right), \\
& n_{c t}=\frac{1}{16}\left(\frac{m}{\sigma}\right)^{2} \frac{\exp \left(-\lambda^{2}\right)}{\operatorname{erfc}\left(\frac{\lambda}{\sqrt{2}}\right)}, \\
& r_{c t}=\sqrt{\frac{8}{\pi}}\left(\frac{\sigma}{m}\right) \exp \left(\frac{\lambda^{2}}{2}\right) \operatorname{erfc}\left(\frac{\lambda}{\sqrt{2}}\right),
\end{aligned}
$$

where

$$
\begin{aligned}
& \sigma=\sqrt{\sigma_{1}^{2}+\sigma_{2}^{2}}, \\
& m=\sqrt{m_{1}^{2}+m_{2}^{2}}, \\
& \lambda=Y / \sigma,
\end{aligned}
$$

where $\sigma_{1}$ and $\sigma_{2}$ are the root mean square (RMS) surface roughness, $m_{1}$ and $m_{2}$ are the mean absolute asperity slope of the contacting surfaces, respectively and $Y$ is the mean plane separation.

A fragment of the joint of the tested bars bed is shown in Fig. 2. Because the bars are characterized by shape errors, the surface contact occurs only on small so-called contour areas, while the major part of the nominal contact surface is occupied by macro-gaps filled with gas. The location and size of the contour area in the analyzed joints are completely accidental. In this case, it causes that thermal contact resistance cannot be determined in an analytical way using models based on Eq. (1). 

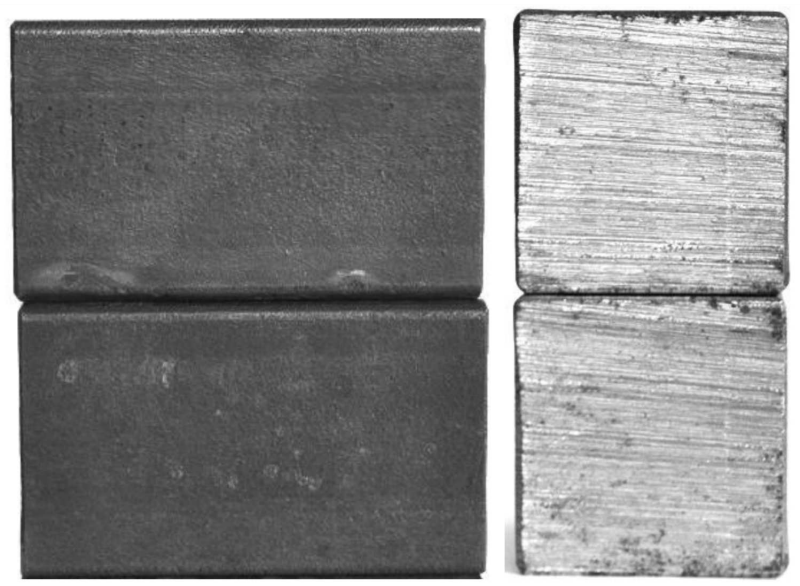

Fig. 2. The view of the fragment of the joint of square bars.

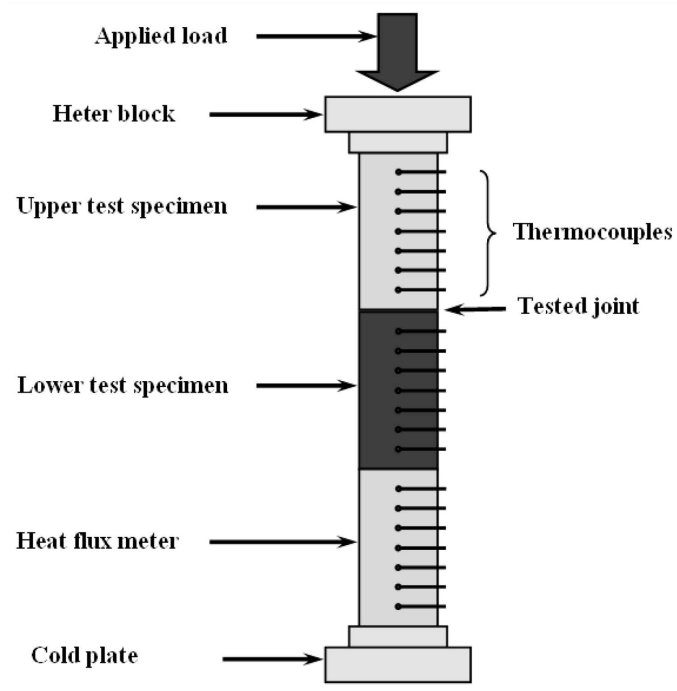

Fig. 3. A typical experimental setup for the determination of thermal contact resistance.

For experimental determination of the value of $R_{c t}$ there are usually used setups like the one show schematically in Fig. 3 [14]. A stack of three samples is placed in the measurement space. Two samples form the test joint, the third sample of known thermal conductivity is used to determine heat flux $q$. Thanks to the appropriate construction of the stand, unidirectional heat flux is generated in these samples. After obtaining a steady state in the system, the temperature distributions in individual samples are determined using set of thermocouples. By a linear extrapolation of the temperature distribution in upper and lower test specimens temperature change is determined within the joint $\Delta t_{j}$. The value of $R_{c t}$ is calculated from relation [14]:

$$
R_{c t}=\frac{\Delta t_{j}}{q} \text {. }
$$

The presented method of measurement cannot be used to determine the thermal resistance of joints formed by bars. This is due to the geometry of these elements. Too small size of the bars does not allow to measure temperature distribution in them using thermocouples. The inability to determine the resistance of $R_{c t}$ for the analyzed bed by analytical and experimental standard methods, prompted the authors to propose an original measurement method, in which a thermovision camera is used to determine the temperature distribution within the tested sample. Thermovision is the second next to the streaking method, an optical visualization technique that allows a broad analysis of heat transfer phenomena in the area of the porous charge $[15,16]$.

In the proposed method, the test bed is placed in a special electric heating chamber. The construction of this chamber ensures obtaining a one-dimensional heat flux in the tested sample. The general view of the described stand is shown in Fig. 4. Figure 4a shows the surface of the bed, the temperature of which is recorded during the experiment. In order to eliminate lateral losses of heat, as shown in Fig. 4a, for the majority of the experiment the tested surface of the bed was covered with a thermal insulation material. This surface was exposed only for a short moment to record a thermogram when a steady state was achieved in the system.
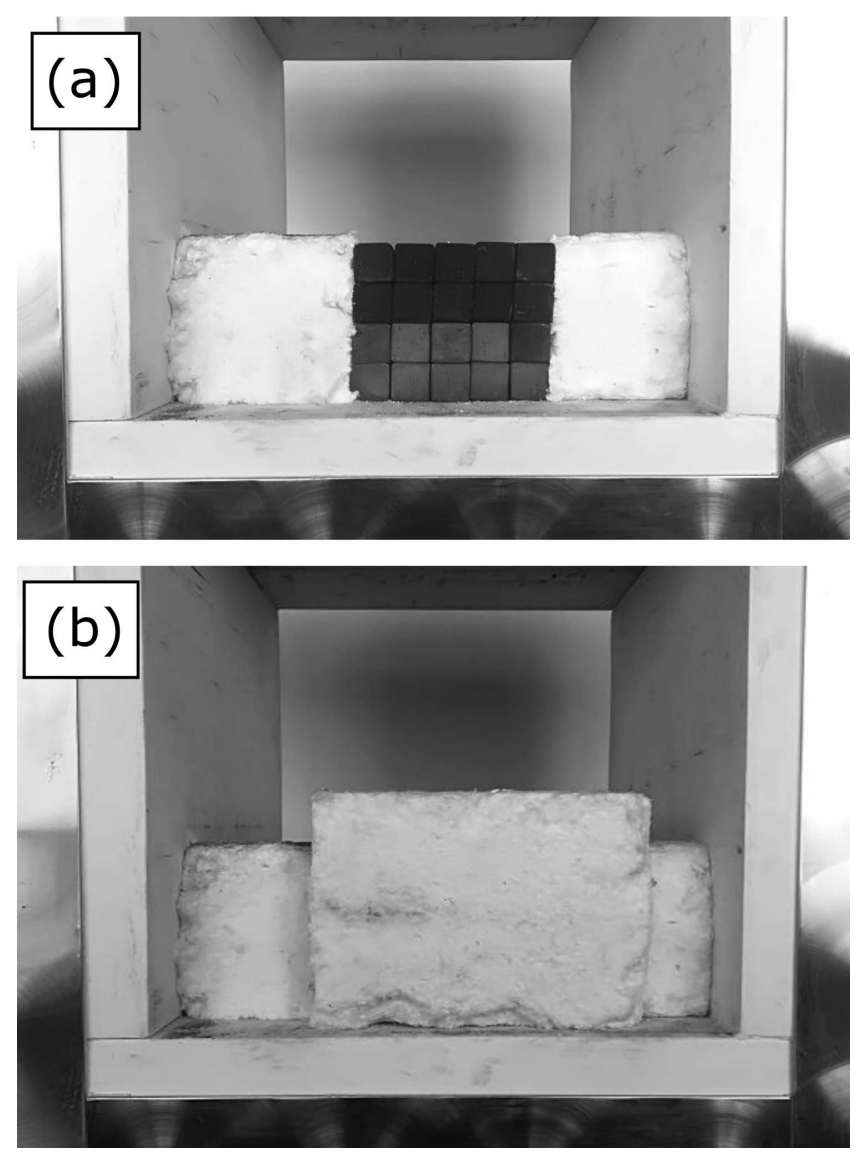

Fig. 4. Experimental setup used for the determination of thermal contact resistance by proposed method: (a) view of the analyzed surface of the tested bed, (b) the area covered by the thermal insulation material to eliminate lateral heat loss. 


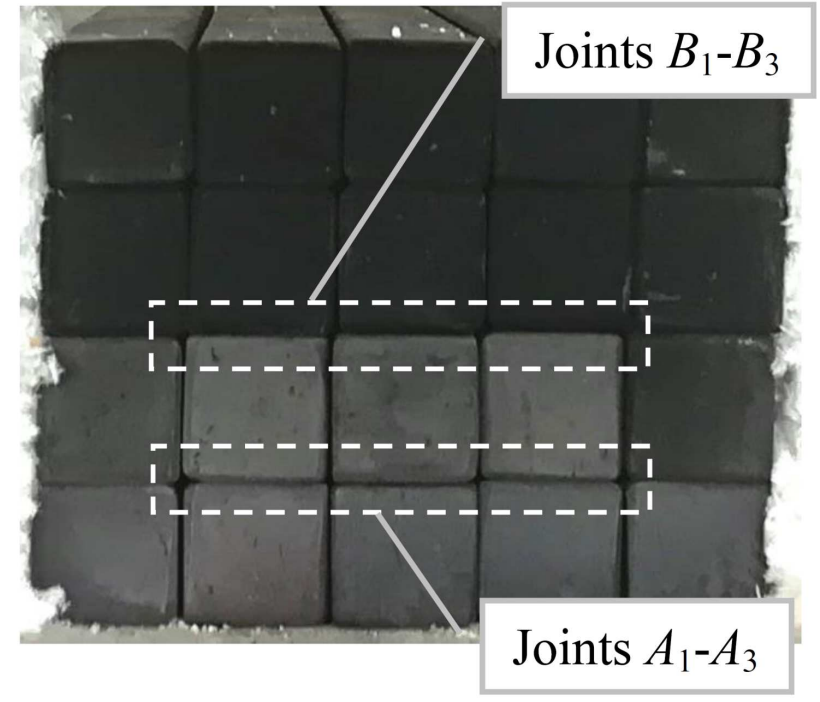

Fig. 5. The view of the bed of tested bars with selection of six analyzed joints.

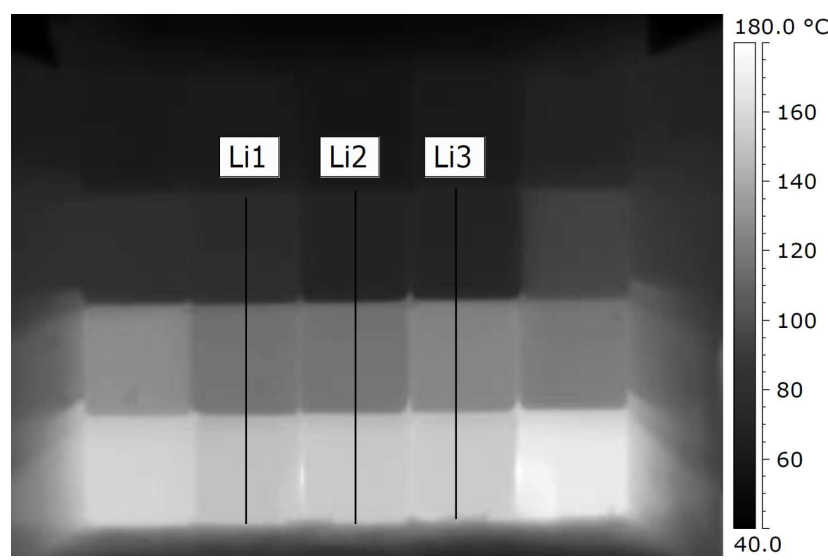

Fig. 6. An example of a thermogram of the bed of tested bars with Li1-Li3 lines.

In the described case, a bed created with four layers of $20 \mathrm{~mm}$ square bars was tested, as shown in Fig. 5 . Figure 6 shows an example of a thermogram on which vertical lines Li1-Li3 are applied, parallel to the direction of the heat flux. The lines run within three layers of bars, along three central rows.

Specialized software allows to develop a graph of the temperature distribution along the lines marked on the thermogram. Such a graph for lines printed on the thermogram from Fig. 6 is shown in Fig. 7. For each temperature distribution there are fragments of a gentle temperature drop $\Delta t_{b r}$ within each of the bars and, between them, rapid temperature faults $\Delta t_{j}$ within the joints. As shown in Fig. 7, the exact values of the mentioned parameters are determined by linear extrapolation of temperature distributions in the cross-section of the bars.

On the basis of the temperature drop $\Delta t_{b r}$ heat flux $q$ is determined in the analyzed bed [14]:

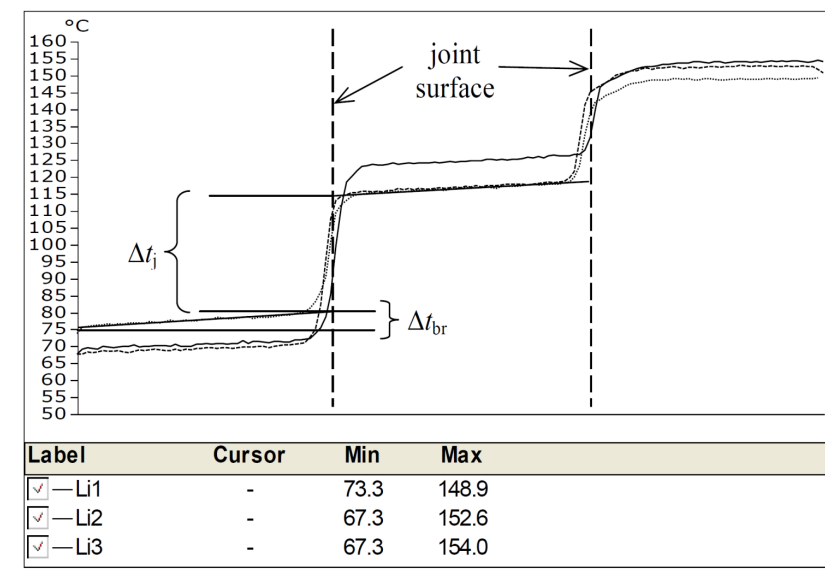

Fig. 7. The temperature distribution along the line Li1-Li3 from thermogram.

$$
q=\frac{k_{b t} \Delta t_{b r}}{a_{b r}}
$$

where $k_{b r}$ - thermal conductivity of the bar, $a_{b r}$ - the transverse dimension of the bar in the direction of the heat flux.

Knowing the heat flux in the sample corresponding to the given thermovision measurement and using the dependence (9), it is possible to determine the $R_{c t}$ value for each joint. This requires only determining the value of $\Delta t_{j}$ corresponding to this joint. Thus, in the proposed research methodology, the temperature distribution in the analyzed medium obtained using a thermovision camera is used to determine the value of thermal resistance of joints.

\section{Results and discussion}

During the research performed for described bed of bars, six thermograms were registered. Each thermogram was recorded for a different heating power, adjustable using an autotransformer plugged into the system of heater's heating. This makes it is possible to determine the value of $R_{c t}$ for a certain temperature range. Because each thermogram should have been registered in steady state (which was obtained after about $3 \mathrm{~h}$ of heating for the tested sample), the measurements were made in two separate turns. The registered thermograms are summarized in Fig. 8. The maximum temperature values obtained within the tested bed for subsequent measurements were as follows: $172^{\circ} \mathrm{C}, 207^{\circ} \mathrm{C}, 297^{\circ} \mathrm{C}, 337^{\circ} \mathrm{C}$, $374{ }^{\circ} \mathrm{C}, 478^{\circ} \mathrm{C}$.

For each thermogram, the temperature distribution along the lines marked in Fig. 6 was determined. The graphs obtained in this way allowed to determine the values of temperature differences $\Delta t_{b r}$ and $\Delta t_{j}$. Having the values of these parameters, using Eqs. (9) and (10), the queried value of resistance of the contact conductivity $R_{c t}$ for selected joints of bed was calculated.

Each of the Li1-Li3 lines plotted in the analysis of thermograms intersect two joints. The lower joints between 

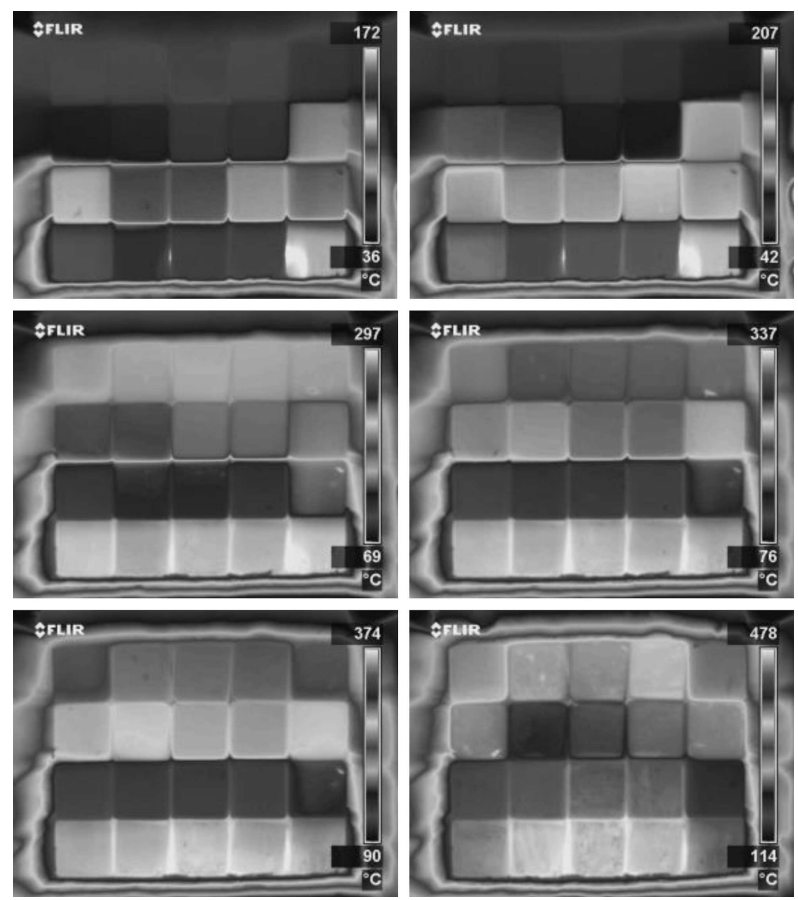

Fig. 8. Summary of subsequent thermograms obtained during the experiment.

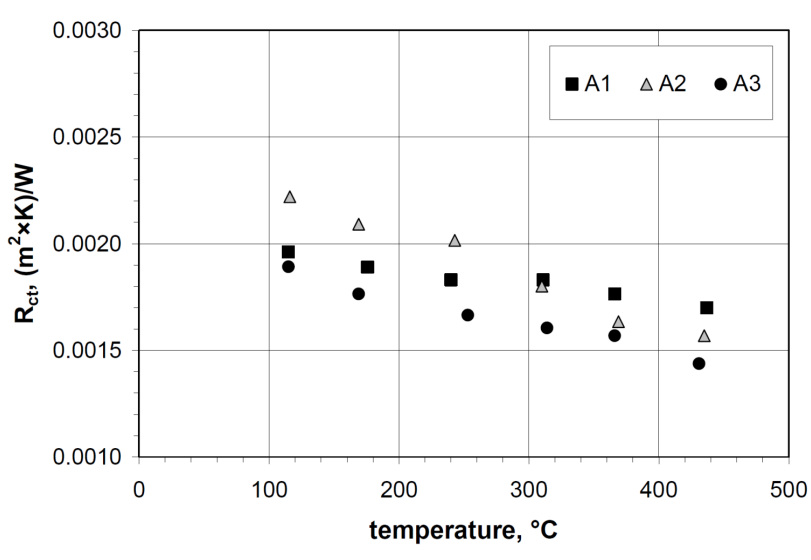

Fig. 9. The value of resistance $R_{c t}$ lower joints in function of temperature.

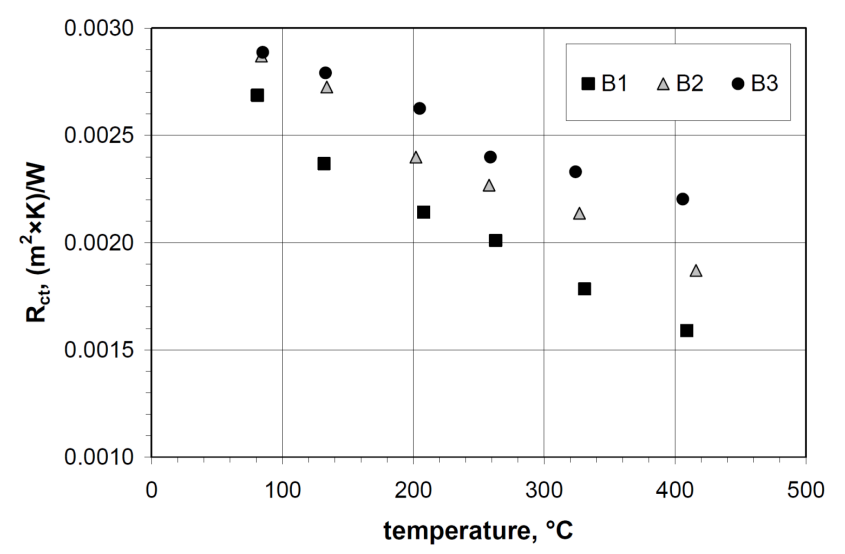

Fig. 10. The value of resistance $R_{c t}$ upper joints in function of temperature. the first and second layer of bars are marked with the letter $A$, while the upper joints between the second and third layer of the letter $B$. In this way, the value of contact resistance of the six joints were determined for the test bed, which were marked with the following symbols: $A 1, A 2, A 3, B 1, B 2, B 3$. These joints are indicated in Fig. 6.

The resistance values $R_{c t}$ obtained for the lower joints are shown in Fig. 9, while for the upper joints in Fig. 10. As can be seen from these graphs, resistance of contact conduction of all six joints decreases as a function of temperature, which is caused by the influence of thermal radiation. This type of heat transfer occurs within the joints in the macro-gap area. As previously mentioned, due to the errors in the shape of the surface of the bars, macro-gaps occupy the majority of the nominal surface of the considered joints. As a result, the proportion of radiation in the total heat transfer occurring within the joints is significant. Since the intensity of this phenomenon depends on the average temperature of the surface of the joint, at higher temperatures the value of $R_{c t}$ decreases. At the same time, for both lower and upper joints at this given temperature a certain dispersion of values is observed. This is due to the individual geometric characteristics of the individual joints.

The resistance of joints $A$ at the lowest temperature $\left(115^{\circ} \mathrm{C}\right)$ is in the range $0.00189 \div 0.00222\left(\mathrm{~m}^{2} \mathrm{~K}\right) / \mathrm{W}$, while at the highest temperature $\left(435^{\circ} \mathrm{C}\right)$ it is $0.00143 \div$ $0.00169\left(\mathrm{~m}^{2} \mathrm{~K}\right) / \mathrm{W}$.

For $B$ joints at the lowest temperature $\left(85^{\circ} \mathrm{C}\right)$ the resistance $R_{c t}$ is in the range $0.00269 \div 0.00280\left(\mathrm{~m}^{2} \mathrm{~K}\right) / \mathrm{W}$, while at the highest temperature $\left(410^{\circ} \mathrm{C}\right)$ it is $0.00159 \div$ $0.00220\left(\mathrm{~m}^{2} \mathrm{~K}\right) / \mathrm{W}$.

From the comparison of the presented data, it can be seen that the lower joints are less resistant than the upper joints. At the same time, the dynamics of temperature changes $R_{c t}$ is greater for upper joints. This observation is easy to interpret. In the lower joints there is more stress than in the upper joints. As it is known, increasing the stress contributes to reducing the thermal resistance of the joints. In addition, the lower the stress, the smaller the coefficient of contact. This, in turn, makes the larger proportion of thermal radiation in the joint. The higher the proportion of radiation, the greater the dynamics of declines of $R_{t c}$ with increase of temperature.

To better determine the difference of $R_{t c}$ values between joints $A$ and $B$, the average value of this parameter was determined for a given temperature. In this way, one value of resistance $R_{c t}$ for joints $A$ and one value for joints $B$ were obtained for each measurement. The values of these resistances are presented in Fig. 11. Then, these results were approximated by linear regression equations, resulting in the following relationships for individual joints:

$$
\begin{aligned}
& R_{c t-A}=-1.39 \times 10^{-6} t+0.00222, \\
& R_{c t-B}=-2.88 \times 10^{-6} t+0.00302 .
\end{aligned}
$$




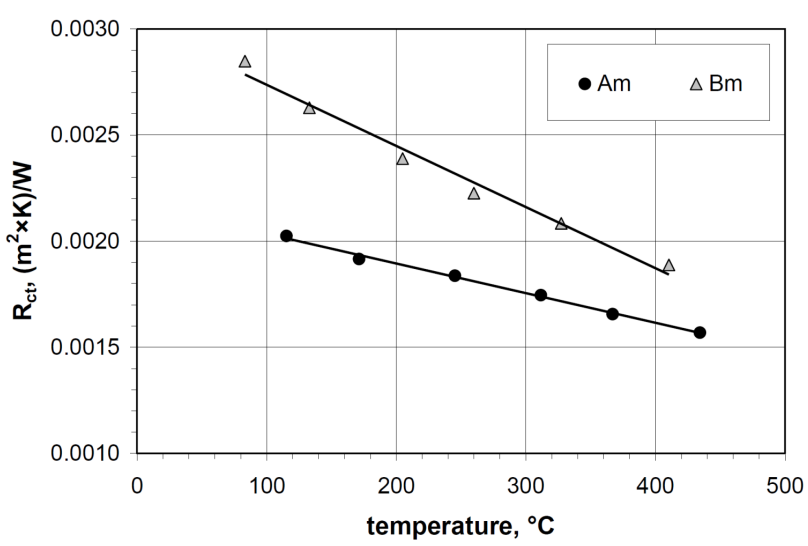

Fig. 11. Averaged values of the resistance $R_{c t}$ of lower and upper joints as a function of temperature.

The values of the coefficient of determination $R^{2}$ of Eqs. (11) and (12) were 0.996 and 0.983 , respectively. Values so close to unity indicate that these dependences are well matched to the average measured values of $R_{c t}$.

Using Eqs. (11) and (12), the relative difference of resistance $R_{c t}$ was calculated for both joints. This parameter defines the following dependence:

$$
\mathrm{d} R_{c t}=\frac{R_{c t-B}-R_{c t-A}}{R_{c t-A}} \times 100 \% \text {. }
$$

Changes in the value of the $\mathrm{d} R_{c t}$ parameter in $50 \div$ $450{ }^{\circ} \mathrm{C}$ temperature range are shown in Fig. 12. As can be seen, the thermal resistance of connectors $B$ is greater than the resistance of joints $A$ from $34 \%$ to $8 \%$ depending on the temperature. The difference in the value of these parameters results from the different contribution of radiation heat transfer within the joints.

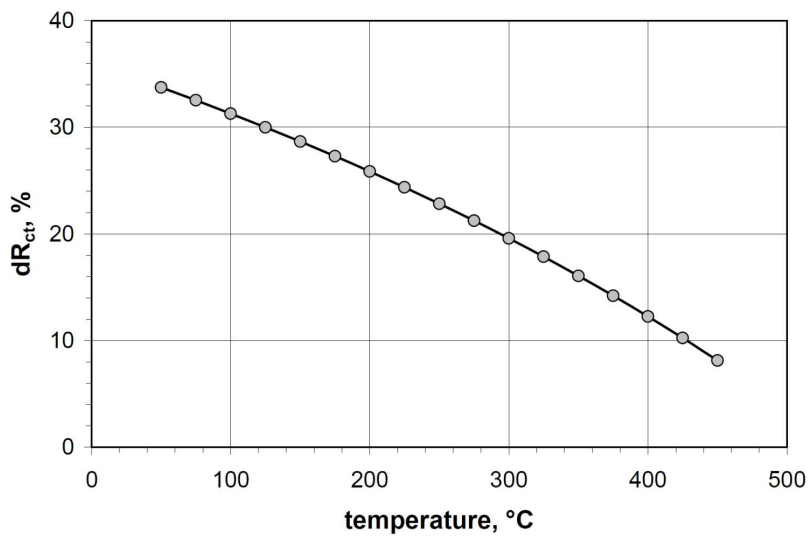

Fig. 12. The value of the parameter $\mathrm{d} R_{c t}$ in function of temperature.

At the end of presented considerations the measurement uncertainty of presented method should be mentioned. To determine this parameter, the uncertainty propagation rule was used [17]. According to this rule, taking into account Eq. (2), the relative uncertainty of resistance $R_{c t}$ was calculated as

$$
\frac{u\left(R_{c t}\right)}{\left|R_{c t}\right|}=\sqrt{\left(\frac{u(q)}{q}\right)^{2}+\left(\frac{u\left(\Delta t_{j}\right)}{\Delta t_{j}}\right)^{2}} .
$$

After taking the measurement uncertainty heat flux $q$ and the temperature difference $\Delta t_{j}$, it was found that in this case the relative uncertainty of the measurement of the resistance $R_{c t}$ is from $4.7 \%$ to $5.3 \%$ depending on the measuring temperature. The result at this level, due to the complexity of the measurement, should be considered acceptable.

\section{Summary and conclusions}

The article presents the original method for measuring of the contact thermal resistance. This method differs from conventional methods by the method of temperature measurement within the elements forming the researched joint. Instead of measuring the contact temperature at several points using a thermocouple system, a non-contact measurement with a thermal camera was used. This makes that it is possible to research joints made of small size elements, where the use of thermocouples is impossible. At the same time, this method is relatively simple to implement. The basic difficulty, as in the implementation of measurements of other thermal parameters, ensures the provision of appropriate boundary conditions within the researched sample. In this case, these conditions were provided by a appropriate design of the heating chamber and the insulation of the lateral surface of the sample. The research was carried out for a sample of a porous charge in the form of a flat bed of square bars. At the same time, the thermal resistance of six joints was analyzed. It was found that the resistance $R_{c t}$ for the researched sample is from $0.00149\left(\mathrm{~m}^{2} \mathrm{~K}\right) / \mathrm{W}$ to $0.00280\left(\mathrm{~m}^{2} \mathrm{~K}\right) / \mathrm{W}$. The increase in temperature and stress causes a noticeable decrease in the value of the $R_{c t}$. The measurement uncertainty of the presented method is about $5 \%$.

\section{References}

[1] M.M. Yovanovich, IEEE Trans. Compon. Packag. Technol. 20, 182 (2005).

[2] P. Furmański, T.S. Wiśniewski, J. Banaszek, Thermal Contact Resistances and Other Thermal Phenomena at Solid-Solid Interface, Institute of Heat Engineering, Warsaw University of Technology, Warsaw 2008.

[3] M. Rywotycki, Archiv. Metall. Mater. 61, 2061 (2016).

[4] M. Rywotycki, Hutnik Wiadomości Hutnicze 83, 13 (2016) (in Polish).

[5] C. Kolmasiak, T. Wyleciał, Metalurgija 57, 4 (2018).

[6] S.S. Sahay, K. Krishman, Iron Steelmak. 34, 89 (2007).

[7] X. Zhang, F. Yu, W. Wu, Y. Zuo, Int. J. Thermophys. 24, 1395 (2003).

[8] M. Kaviany, Principles of Heat Transfer in Porous Media, 2nd ed., Springer-Verlag, New York 1995.

[9] N. Wakao, K. Wato, J. Chem. Eng. 2, 24 (1968). 
[10] R. Wyczolkowski, D. Urbaniak, J. Thermophys. Heat Transfer 30, 721 (2016).

[11] B.B. Mikic, Int. J. Heat Mass Transfer 17, 205 (1974).

[12] M.G. Cooper, B.B. Mikic, M.M. Yovanovich, Int. J. Heat Mass Transfer 12, 297 (1969).

[13] M.R. Sridhar, M.M. Yovanovich, J. Thermophys. Heat Transfer 8, 633 (1994).

[14] Y.A. Cengel, Heat and Mass Transfer - A Practical Approach, 3rd ed., McGraw-Hill, New York 2007.
[15] R. Wyczółkowski, C. Kolmasiak, D. Urbaniak, T. Wyleciał, Archiv. Metall. Mater. 60, 2949 (2015).

[16] R. Wyczolkowski, D. Musiał, Exp. Therm. Fluid Sci. 51, 122 (2013).

[17] J.R. Taylor, An Introduction to Terror Analysis. The Study of Uncertainties in Physical Measurements, Oxford University Press, Oxford 1982. 